PAPER

Modeling and fabrication of electrostatically actuated diaphragms for onchip valving of MEMS-compatible microfluidic systems

To cite this article: Ali Can Atik et al 2020 J. Micromech. Microeng. 30115001

View the article online for updates and enhancements.

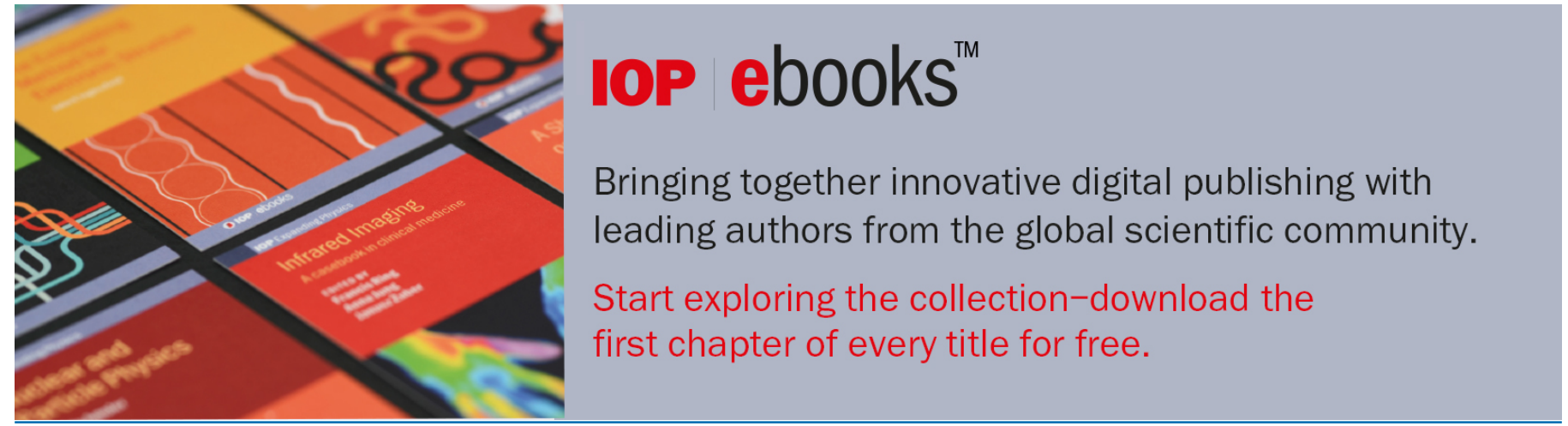

This content was downloaded from IP address 144.122 .90 .199 on 10/11/2020 at 13:40 


\title{
Modeling and fabrication of electrostatically actuated diaphragms for on-chip valving of MEMS-compatible microfluidic systems
}

\author{
Ali Can Atik ${ }^{1,2}$, Metin Dündar Özkan ${ }^{1,2}$, Ebru Özgür ${ }^{2}$ (D) , Haluk Külah ${ }^{1,2}$ (D) \\ and Ender Yıldırım ${ }^{2,3}$ (D) \\ ${ }^{1}$ Department of Electrical and Electronics Engineering, Middle East Technical University, Ankara, \\ Turkey \\ ${ }^{2}$ METU MEMS Research and Application Center, Ankara, Turkey \\ 3 Department of Mechanical Engineering, Middle East Technical University, Ankara, Turkey \\ E-mail: yender@metu.edu.tr
}

Received 25 February 2020, revised 19 June 2020

Accepted for publication 30 June 2020

Published 14 July 2020

\begin{abstract}
This paper presents an analytical model to estimate the actuation potential of an electrostatic parylene-C diaphragm, processed on a glass wafer using standard microelectromechanical systems (MEMS) process technology, and integrable to polydimethylsiloxane (PDMS) based lab-on-a-chip systems to construct a normally-closed microvalve for flow manipulation. The accurate estimation of the pull-in voltage of the diaphragm is critical to preserve the feasibility of integration. Thus, we introduced an analytical model, in a good agreement with the finite element method (FEM), to extend the solution of the pull-in instability by including the effect of nonlinear stretching for multilayered circular diaphragms. We characterized the operation of fabricated diaphragms with a $300 \mu \mathrm{m}$ radius for the parameters, including pull-in voltage (221 V on average), opening and closing response times (in microseconds), repeatability (more than 50 times $)$, and touch area $(25.3 \% \pm 2.6 \%$ at pull-in potential). The experimental pull-in voltage shows close accuracy with the predicted results. Moreover, the diaphragm, sealed with a PDMS microchannel, was tested under fluid flow to prove the applicability of microfluidic integration. The hybrid fabrication method enables the realization of optically transparent and durable electrostatic microvalves for complex functioning of polymer-based microfluidic systems, as the extended analytical formulation permits accurate modeling of operation.
\end{abstract}

Supplementary material for this article is available online

Keywords: circular diaphragm, electrostatic actuation, microvalve, MEMS

(Some figures may appear in colour only in the online journal)

\section{List of symbols}

a

$t_{i}$

$g$

$V_{d c}$

$E_{i}$ diaphragm radius $(\mathrm{m})$

thickness of the $i$ th plate $(\mathrm{m})$

the initial air gap (m)

DC voltage applied between the top and bottom electrodes (V)

elastic modulus of the $i$ th plate (GPa) $v_{i}$

$\varepsilon_{p}$

$\varepsilon_{\text {air }}$

$\varepsilon_{0}$

$n$

$F_{m}$

$F_{e}$

$q$
Poisson's ratio of the $i$ th plate dielectric constant of parylene-C dielectric constant of air vacuum permittivity $\left(\mathrm{F} \mathrm{m}^{-1}\right)$ number of the plies in the diaphragm mechanical restoring force $(\mathrm{N})$ electrostatic attraction force $(\mathrm{N})$ uniform load $(\mathrm{Pa})$ 


$\begin{array}{ll}w & \text { displacement }(\mathrm{m}) \\ w_{\text {peak }} & \text { peak displacement }(\mathrm{m}) \\ w_{\text {avg }} & \text { average displacement }(\mathrm{m}) \\ k_{1} & \text { linear spring constant }\left(\mathrm{N} \mathrm{m}^{-1}\right) \\ k_{3} & \text { cubic spring constant }\left(\mathrm{N} \mathrm{m}^{-3}\right) \\ D_{i} & \text { flexural rigidity of the } i \text { th plate }\left(\mathrm{N} \mathrm{m}^{2}\right) \\ C & \text { electrical capacitance }(\mathrm{F}) \\ r & \text { distance between the center and any point on } \\ & \text { the diaphragm }(\mathrm{m}) \\ U_{e} & \text { electrostatic potential energy }(\mathrm{J}) \\ V_{P I} & \text { pull-in voltage }(\mathrm{V}) \\ w_{\text {avg,PI }} & \text { average displacement at pull-in voltage }(\mathrm{m}) \\ w_{\text {peak,PI }} & \text { peak displacement at pull-in voltage }(\mathrm{m})\end{array}$

\section{Introduction}

As the technology evolves, and the expectations change for chemical and biological analyses, the researchers have been endeavoring to go beyond the capabilities of what was firstly introduced by Manz et al [1], the micro total analysis systems ( $\mu$ TAS). The efforts put forward for more precise and faster results at lower costs, and more automated operation at the micro-level pave the way for new technologies and applications. The internal or external use of sensors, pumps, and valves in microfluidic systems contributes to these efforts by yielding a more functional system. Microvalves are one of the essential microfluidic components, which are employed as on/off switches along microchannels to deliver complex functionality.

Mechanical active microvalves can adopt several actuation methodologies to accomplish the movement of the mechanical part, usually in the form of a flexible diaphragm. These methods mainly include magnetic [2-8], electrostatic [9-14], piezoelectric [15-19], thermal [20-24] and pneumatic actuation [25-30]. Among these, microvalves operated by pneumatic actuation are useful in LOC applications due to their excellent rapid switching performance, versatility, and simple fabrication compatible with elastomeric substrates such as polydimethylsiloxane (PDMS) [31, 32]. They also enable complex manipulation of the flow and no leakage behavior because high-pressure input can be applied [30]. However, the requirement of additional bulky off-chip components such as external pressure sources, solenoid microvalves, and their connections is a drawback restricting the compactness of the system, which is incompatible with the miniaturization and portability principles of LOC systems. In this regard, the electrostatic actuation scheme, which needs less bulky off-chip components regarding power electronics, receives considerable attention. Here, the integration of application-specific integrated circuits (ASICs) designed to drive high voltage for electrostatic actuators can enable the system-level miniaturization [33].

Traditionally, electrostatic microvalves incorporate a mechanical moving part in the form of a flexible diaphragm to manipulate the flow inside a microchannel. Thus, estimating the pull-in voltage of diaphragm actuators is critical to determine the operating voltages of microvalves relying on electrostatic actuation. A well-known parallel plate capacitor model renders inaccurate results mainly because it ignores the nonlinear deflection and fixed boundary condition at the periphery of the diaphragm. Previously, numerical and analytical models to account for the nonlinear deflection of electrostatically actuated microstructures have been developed [34-37]. However, these models ignore the effect of the electrode coating on the definition of the total diaphragm stiffness. Here, we present an extended analytical model that accurately describes the pullin behavior of the multilayered diaphragm to guide the consequent design of the electrostatic microvalve.

On the other hand, the implementation of electrostatic actuators into a LOC system might introduce serious issues such as the possibility of electrolysis of working fluids, electrode polarization, and electrical double layer formation [38]. These scenarios can be eliminated by the application of highfrequency AC signals [39], and electrode passivation to obviate the direct contact between the electrode and working fluid [38].

Previously, we have reported an in-plane normally-closed electrostatic microvalve for use in parylene-based LOC systems [40]. The proposed design isolates the working fluid from the electric field by placing the diaphragm underneath the microchannel, thereby offering a reliable actuation mechanism for wet applications. However, since it was built on a silicon substrate, the microvalve was not optically transparent, which is demanded by many of the LOC applications. Moreover, the utilization of parylene as the channel material hinders the durability of the design. Tice et al adapted a PDMS-based fabrication process, including replica molding, micro-transfer printing, and plasma bonding, to implement a similar microvalve [11], which solves the problems related to optical transparency and durability. On the other hand, many LOC applications require functional elements such as electrodes for sensing, which are not convenient to integrate with all-PDMS devices. In these applications, standard MEMS fabrication methods are more amenable to produce the electrodes reliably.

In this paper, we also propose a new hybrid fabrication scheme to produce a normally-closed electrostatic microvalve comprising a circular diaphragm with an embedded electrode. The active components of the microvalve are fabricated on a glass substrate by MEMS fabrication techniques and integrated with a PDMS microchannel. As a result, the PDMS-glass structure provides both transparency and durability, while MEMS fabrication allows the implementation of functional elements. In the following sections, we describe the structure of the normally-closed electrostatic microvalve, present the detailed analytical model and numerical verification of it, fabrication of the microvalve, and its experimental characterization.

\section{Design}

Figure 1(a) illustrates the conceptual cross-sectional view of the valve structure, structural materials, and design dimensions of the electrostatically actuated diaphragm. The valve comprises a layered circular diaphragm clamped at its periphery 

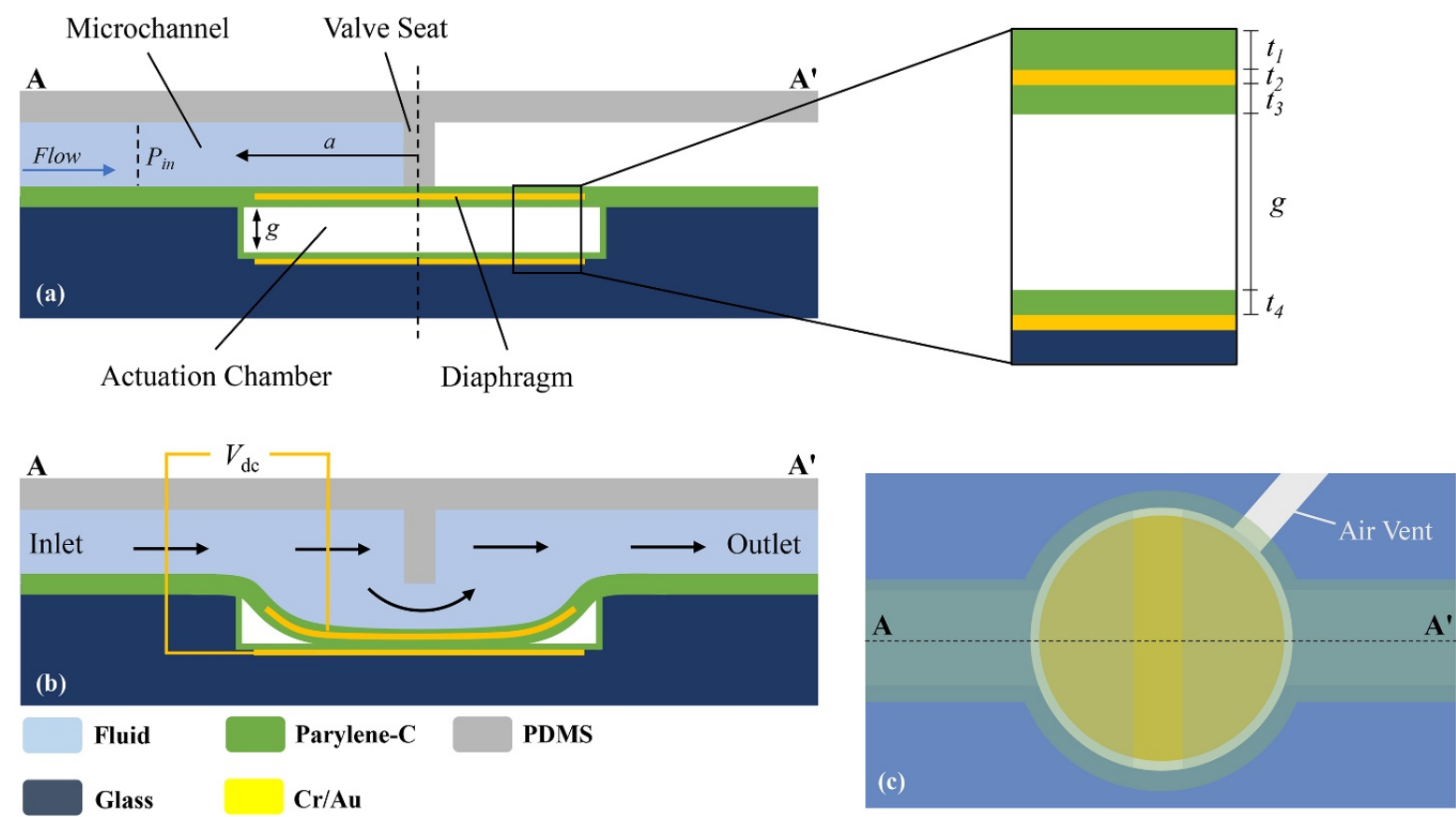

Figure 1. The structure, design, and operation states of the electrostatic microvalve. (a) In closed state (above), the liquid flow is prevented by the wall completely covering the cross-sectional area of the microchannel. (b) When a specific voltage is applied, the diaphragm collapses on the bottom electrode and enables flow in the open state (below). (c) The top view of the microvalve.

with a radius $a$. We preferred parylene-C, a polymer compatible with MEMS microfabrication, as the structural material for both elastic (Young's modulus of $3.2 \mathrm{GPa}$ ) and dielectric properties (relative permittivity of 3.15). Parylene-C is a promising candidate for electrostatic-driven diaphragm definition because its soft polymeric nature can reduce the required voltage for actuation while providing electrical insulation between conductive layers. A thin gold layer of thickness $t_{2}$, embedded between the structural parylene layer and the insulation layer with respective thicknesses $t_{1}$ and $t_{3}$, defines the top electrode. Underneath the diaphragm, there exists an actuation chamber where a venting channel connects it to ambient pressure (figure 1(c)). At the base of the actuation chamber another gold layer forms the fixed bottom electrode. The height of the chamber $(g)$ defines the initial air gap between the diaphragm and the bottom of the actuation chamber. A thin parylene $\left(t_{4}\right)$ on the bottom electrode reduces the adhesion of the diaphragm during its release, as explained in the fabrication section.

A valve seat, formed in a flat rectangular shape protruding from the inner top wall of PDMS microchannel, lies in the center of the diaphragm. At the closed state, this wall prevents the flow along the microchannel. A DC voltage $\left(V_{\mathrm{dc}}\right)$, applied across the bottom and top electrodes, induces an electrostatic force on the diaphragm. The electrostatic force excites a movement by pulling the diaphragm towards the fixed bottom electrode. As expected, the diaphragm deflects more while increasing the voltage. At pull-in, it collapses on the base of the actuation chamber and permits the flow under the wall. In the open state, the air inside the actuation chamber can escape through the venting channels. As the applied voltage increases further after pull-in, the area, where the diaphragm touches the base, expands. The schematic in figure 1(b) illustrates the conceptual cross-sectional view of the valve in the open state.

The channel height of normally-open electrostatic microvalves defines the gap between electrodes and can incur low actuation forces in response to the driving voltage. In order to circumvent this problem, in normally-closed architecture, the diaphragm resides under the fluid channel, and the valve seat blocks the flow. Thus, a deflection in the range of few microns, independent of the channel height, will be enough to allow the fluid to pass underneath the valve seat during operation. Placing the diaphragm underneath the channel also enables a reliable actuation mechanism for wet microfluidic applications by avoiding the electrolysis of fluid samples. Furthermore, the electrical power will be consumed for a short time to change the state of the valve. There will be no current flow, so no power consumption due to the capacitive steady-state behavior in the open state.

The presented microvalve entails an in-plane parylene diaphragm permitting the flow under the wall when actuated. We define actuation voltage as the minimum required potential, where the deflection of the diaphragm underneath the center of the valve seat is at its maximum. The center of the diaphragm reaches its maximum deflection at pull-in voltage. Thus, a methodical formulation is critical to capture the interplay between mechanical restoring force and electrical force to accurately determine the operating voltages of electrostatic diaphragm actuators.

The operating voltage of the diaphragm depends on the top electrode $\left(t_{2}\right)$ and parylene thicknesses $\left(t_{1}, t_{3}\right.$, and $\left.t_{4}\right)$, diaphragm diameter $(a)$, chamber depth $(g)$, mechanical, and electrical material properties. The geometric design parameters, mechanical and electrical properties of materials, involved 
Table 1. Parameters and material properties involved in the design of electrostatic diaphragm.

\begin{tabular}{|c|c|c|}
\hline Design Dimensions & Mechanical Properties & Electrical Properties \\
\hline Diaphragm radius: $(a=300 \mu \mathrm{m})$ & $\begin{array}{l}\text { Elastic modulus of parylene: }\left(E_{p}=3.2\right. \\
\text { GPa) }\end{array}$ & Dielectric constant of parylene: $\left(\varepsilon_{p}=3.15\right)$ \\
\hline Gap: $(g=5$ to $15 \mu \mathrm{m})$ & Elastic modulus of gold: $\left(E_{A u}=70 \mathrm{GPa}\right)$ & Dielectric constant of air: $\left(\varepsilon_{\text {air }}=1\right)$ \\
\hline Top electrode thickness: $\left(t_{2}=0.2 \mu \mathrm{m}\right)$ & Poisson's ratio of parylene: $\left(v_{p}=0.33\right)$ & $\begin{array}{l}\text { Vacuum permittivity: } \\
\left(\varepsilon_{0}=8.85 \times 10^{-12} \mathrm{~F} \mathrm{~m}^{-1}\right)\end{array}$ \\
\hline $\begin{array}{l}\text { Parylene thicknesses: }\left(t_{1}, t_{4}\right)=(1 \mu \mathrm{m} \text {, } \\
0.75 \mu \mathrm{m})\left(t_{3}=1 \text { to } 20 \mu \mathrm{m}\right)\end{array}$ & Poisson's ratio of gold: $\left(v_{A u}=0.44\right)$ & \\
\hline
\end{tabular}

in the analytical and numerical analysis of electrostatically actuated diaphragm, are listed in table 1 . We selected the diaphragm radius as $300 \mu \mathrm{m}$. Since they contribute effectively to both electrical and mechanical forces, diaphragm thickness $\left(t_{3}\right)$ and chamber depth $(g)$ were selected as significant geometric design parameters and varied intending to minimize actuation potential while maintaining the feasibility of the design. The thicknesses of the insulation $\left(t_{1}\right)$, adhesion $\left(t_{4}\right)$, and top electrode layers $\left(t_{2}\right)$ were kept constant.

\section{Modeling of electrostatically actuated diaphragm}

The large deflection of clamped circular plates was studied to characterize the behavior of the diaphragm under an applied DC voltage. If the deflection is small compared to the plate thickness, it is safe to neglect in-plane strains in the middle surface in comparison with the strains due to bending. For the case in which the deflection is on the order of plate thickness but still small when compared with the diameter, the diaphragm begins to stretch where in-plane strains are no longer of a negligible order of magnitude [41]. Hence, the calculations should be advanced to cover the middle surface strain to obtain more realistic behavior of the diaphragm under applied electrostatic pressure. Moreover, despite being much thinner $(200 \mathrm{~nm})$ than structural parylene-C layers, the contribution to the stiffness of the diaphragm due to the electrode layer can be comparable due to its higher elastic modulus (70 GPa). Here, we extended the analytical model to include the large deflection analysis of a multilayered composite circular diaphragm, which consists of $n$ plies.

The static equilibrium condition (1) yields the deflection of the suspended electrode where the mechanical elastic force $\left(F_{m}\right)$ equals the electrostatic attraction force $\left(F_{e}\right)$ acting on the diaphragm. Additionally, another force applied to the unit area of the diaphragm is the fluidic pressure. This pressure tends to move the diaphragm down, although the microvalve is in the closed state. While expected that fluidic pressure reduces the actuation voltage, its non-uniform nature obstructs the simplicity of the analytical model. Numerical methods must be employed to reveal the relationship between diaphragm deflection and fluidic pressure. Thus, the model estimates the pull-in of the electrostatic diaphragm in the absence of fluid flow as the worst-case operational scenario in this paper.

$$
F_{m}-F_{e}=0
$$

An electrostatic actuator typically yields two possible equilibrium deflections where the first derivative of total potential energy with respect to deflection is zero, which means that the net force on the diaphragm is zero. Here, if the second derivate of the stored energy at an equilibrium point is larger than zero, this point is a local minimum, and the system is stable. However, if it is smaller than zero, the system is in an unstable equilibrium state. The pull-in occurs when the second derivative of the stored energy equals zero at some value of applied voltage, where two points of equilibrium merge at an inflection point [42]. Beyond pull-in voltage, the diaphragm collapses because no equilibrium state exists, and any perturbation to the system will induce an electrostatic attraction force greater than the mechanical restoring force $\left(F_{e}>F_{m}\right)$. Supplementary Material (available at stacks.iop.org/JMM/30/115001/mmedia) includes the details of the analytical model for pull-in estimation and the FEM simulations included in the following sections.

\subsection{Estimation of mechanical restoring force}

Equation (2) represents the general form of the restoring mechanical force for the diaphragm of radius $a$ under uniform load $(q)$, where $k_{1}$ and $k_{3}$ are linear and cubic spring constants of the system and $w_{\text {avg }}$ is equal to one-third of the peak displacement $\left(w_{\text {peak }}\right)$, which occurs at the center [37].

$$
F_{m}=q \pi a^{2}=k_{1} w_{a v g}+k_{3} w_{a v g}^{3}
$$

In order to take the gold electrode into account, we interpreted the diaphragm as a laminated composite plate, which consists of three plies. Since the diaphragms of the electrostatic actuator are non-symmetric through its thickness, the plane of zero strain does not coincide with the geometric mid-plane and should be adjusted. We assume that the curvatures are the same for all plies because they are perfectly intact, and the plate thickness does not change after deformation. For symmetrical bending of a uniformly loaded circular plate of $n$ layers, the coordinate of the neutral plane (3) was approximated by assuming linearly varying strain distribution through the thickness, where $E_{i}, v_{i}$, and $t_{i}$ are elastic modulus, Poisson's ratio, and the thickness of the $i$ th plate, and $t_{j}$ is the thickness of the $j$ th plate.

$$
d=\frac{\sum_{i=1}^{n} \frac{E_{i} t_{i}}{\left(1-v_{i}^{2}\right)} \sum_{j=i}^{n} t_{j}-\sum_{i=1}^{n} \frac{E_{i} t_{i}}{\left(1-v_{i}^{2}\right)} \sum_{j=1}^{n} t_{j}}{2 \sum_{i=1}^{n} \frac{E_{i} t_{i}}{\left(1-v_{i}^{2}\right)}}
$$



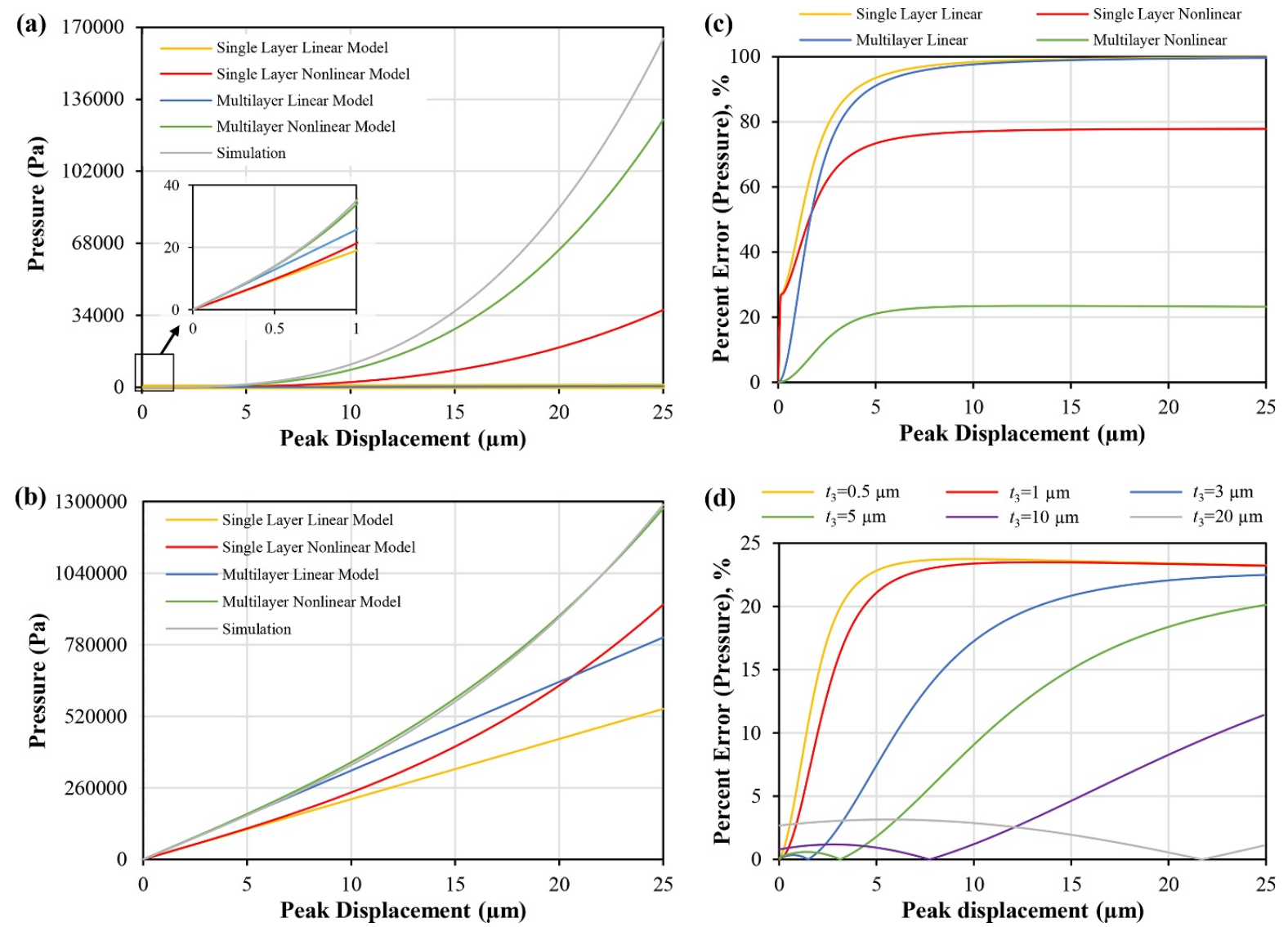

Figure 2. Peak displacements for applied uniform pressure values. (a) $t_{3}=1 \mu \mathrm{m}$. The inset plot illustrates where the diaphragm is inclined to develop relatively large stresses, resulting in nonlinearity in response to pressure at a predefined peak displacement up to the plate thickness. (b) $t_{3}=20 \mu \mathrm{m}$. (c) Prediction errors for different analytical models. (d) Prediction errors for different diaphragm thicknesses $\left(t_{3}\right)$ computed for the nonlinear model for multilayer plates.

For a clamped circular plate with the boundary condition of clamped edges under a uniform load, a useful formulation was obtained to estimate the spring constants by applying the energy method. The total strain energy of the plate including the effects of both bending and stretching can be employed to derive large deflections by using the principle of virtual displacements, where total work exerted by external forces should be equal to the corresponding change in strain energy for assumed infinitely small variation in displacement [41]. From this principle, linear (4) and cubic (5) spring constants of a multilayered plate of $n$ plies can be expressed by following general formulations where $E_{i}, v_{i}, D_{i}$, and $t_{i}$ are elastic modulus, Poisson's ratio, flexural rigidity and thickness of the $i$ th plate, $b_{i-1}$ is the distance between the bottom of the $i$ th plate and the neutral plane $(d)$, and $b_{i}$ is the distance between the top of the $i$ th plate and the neutral plane $(d)$ :

$$
\begin{gathered}
k_{1}=\frac{64 \pi}{a^{2}} \sum_{i=1}^{n} \frac{E_{i}\left(b_{i}^{3}-b_{i-1}^{3}\right)}{1-v_{i}^{2}} \\
k_{3}=\frac{81 \pi}{a^{2}}\left(\frac{-2109 v_{m}^{2}+3210 v_{m}+5679}{625}\right) \frac{\sum_{i=1}^{n} D_{i} \frac{\prod_{i=1}^{n} t_{i}^{2}}{t_{i}^{2}}}{\prod_{i=1}^{n} t_{i}^{2}} \text { and }
\end{gathered}
$$

We computed the solution of the analytical models by using MATLAB for parameters in table 1. The linear and singlelayer models exclude the nonlinear spring constant and the existence of the electrode, respectively. The finite element simulation was employed as a second tool for modeling the electrostatic diaphragm to obtain numerical results, which were considered as the exact values of mechanical restoring force. Numerical simulations were conducted using Solid Mechanics Interface in COMSOL Multiphysics ${ }^{\circledR}$ 5.3a to validate the applicability of the analytical approximation. The 2D axisymmetric model was used as a computational domain to reduce the solution time of simulations. To corroborate the accuracy of the mechanical model for nonlinear deflection behavior of layered diaphragms, we obtained the required pressures to satisfy predefined peak displacement values by both the analytical model and FEM simulation. We selected $0.5 \mu \mathrm{m}$ and $20 \mu \mathrm{m}$ as two extreme values of the thickness $\left(t_{3}\right)$ for the parametric study, and the quantities were computed for a maximum diaphragm displacement of $25 \mu \mathrm{m}$. 

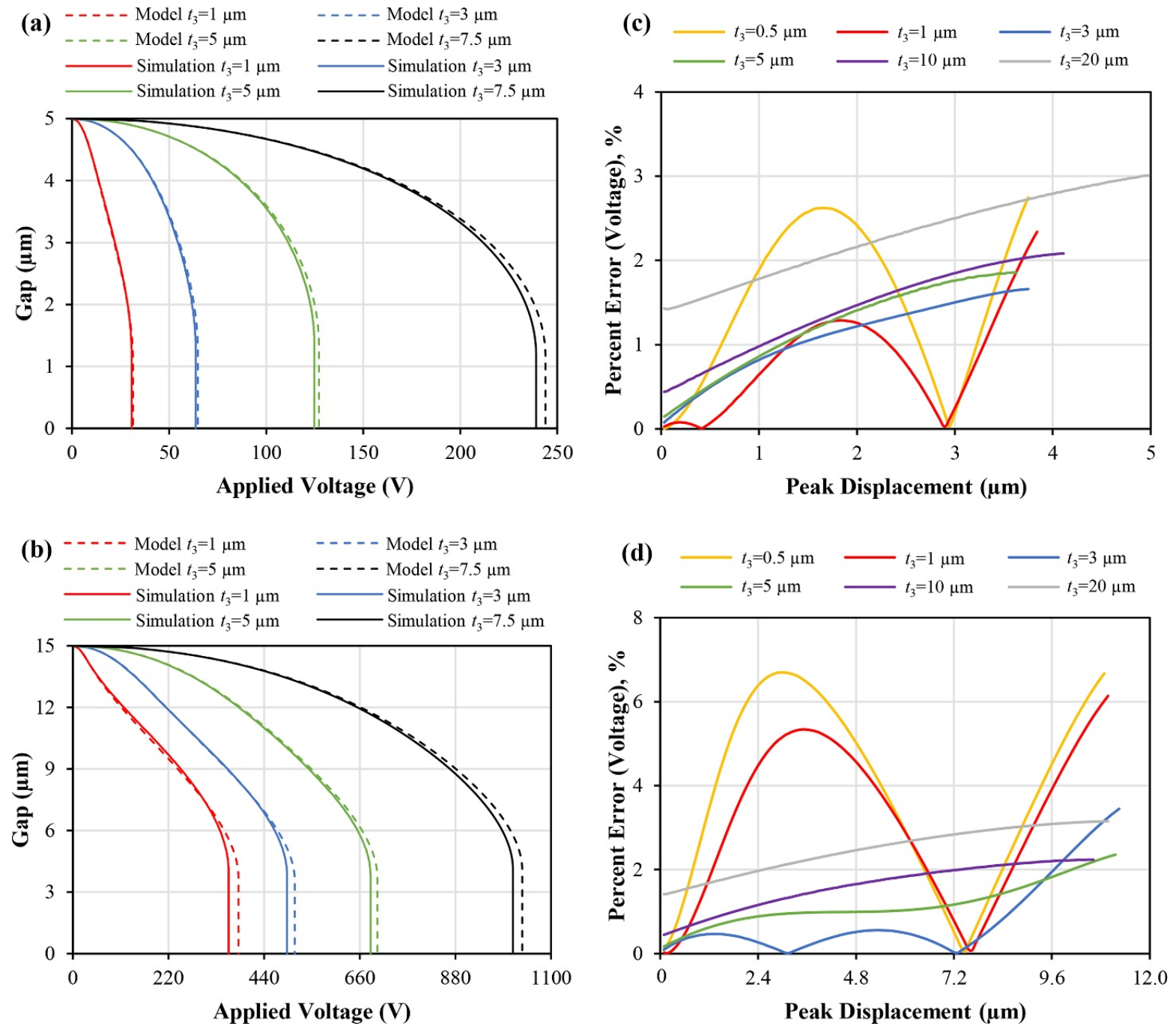

Figure 3. Obtained voltage and displacement values by nonlinear model for multilayer plates and COMSOL simulations for a diaphragm of variable thickness ( $\left.t_{3}\right)$ with a $300 \mu$ m radius. (a) $g=5 \mu \mathrm{m}$. (b) $g=15 \mu \mathrm{m}$. (c) Prediction errors for different analytical models. (d) Prediction errors for different diaphragm thicknesses $\left(t_{3}\right)$ computed for the nonlinear model for multilayer plates.

Figures 2(a) and (b) show the necessary pressure values applied to obtain a center deflection up to $25 \mu \mathrm{m}$ for different diaphragm thicknesses $\left(t_{3}\right)$. When nonlinearity arising from the stretching of the diaphragm was ignored, the deflection increased linearly with applied pressure, so the linear model cannot quite capture the numerically calculated correct movement, which is more apparent for a thinner plate. Nonlinear effects especially became apparent, where the resulting peak displacement is comparable with the diaphragm thickness. Moreover, the inclusion of a thin metal layer results in a lower displacement value for the same applied pressure.

We define a prediction error, which expresses the discrepancy between the pressure values obtained by simulation and approximated by analytical models at a predefined center displacement. The percent error was calculated by the absolute difference between the numeric and approximate analytical solutions, divided by the numeric value, and multiplied by $100 \%$. As illustrated in figure 2(c), with the addition of nonlinear spring constant $\left(k_{3}\right)$ and the electrode layer, the multilayer nonlinear model analytically captured the mechanical behavior more accurately with a percent error below $25 \%$, which is significantly better than other approximations, for $t_{3}=1 \mu \mathrm{m}$. The exclusion of the electrode layer causes a sudden jump in the error plot at the first increment of the peak displacement $(0.125 \mu \mathrm{m})$. Furthermore, the percent error further decreases, as shown in figure $2(\mathrm{~d})$, while increasing diaphragm thickness $\left(t_{3}\right)$. Thus, the nonlinear analytical model for multilayer plate dynamics is the best estimation for diaphragm deflection.

\subsection{Formulation of electrical force and pull-in voltage}

In order to obtain the electrostatic force acting on the diaphragm, the electrical capacitance (6) between the moving top and fixed bottom electrodes should be calculated by including the shape function of the deflection and dielectric parylene layers.

$$
C=\int_{0}^{a} \frac{2 \pi r \varepsilon_{0}}{h-3 w_{\text {avg }}\left(1-\frac{r^{2}}{a^{2}}\right)^{2}} d r=\varepsilon_{0} \pi a^{2} \frac{\operatorname{arctanh}\left(\sqrt{3 w_{\text {avg }} / h}\right)}{\sqrt{3 w_{\text {avg }} h}},
$$




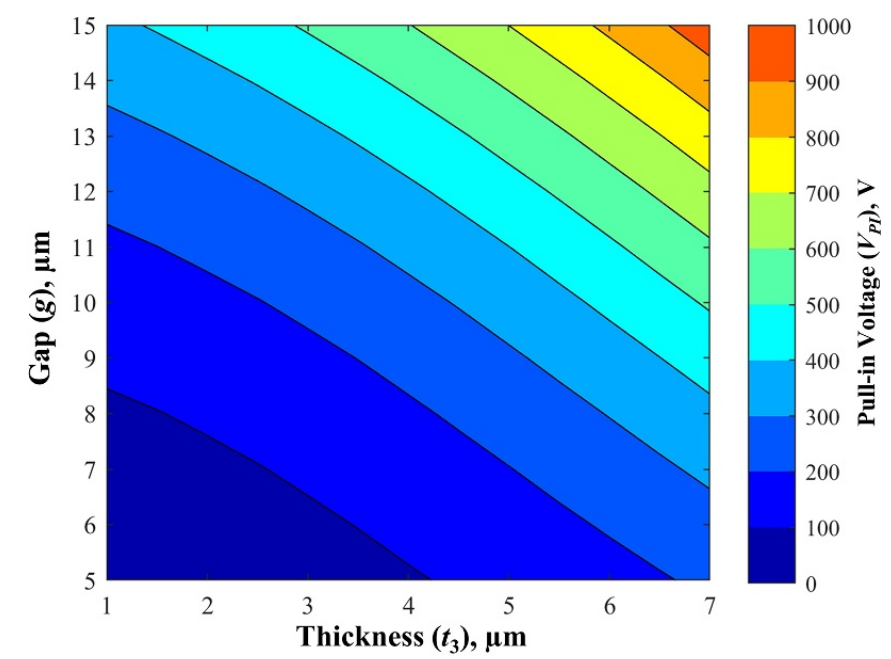

Figure 4. Contour plot of pull-in voltage estimation for diaphragm actuator design with a $300 \mu \mathrm{m}$ radius.

$$
\text { where } h=g+\frac{\left(t_{3}+t_{4}\right)}{\varepsilon_{p}}
$$

For the proposed actuator, fringing fields, which are peripheral electric fields outside the volume between two parallel plates, make an insignificant contribution to the capacitance, because the electrode spacing is much smaller compared to the area of the diaphragm. Thus, the effects of fringing fields were excluded from formulation. Finally, the electrical force (7) acting on the diaphragm due to the applied voltage can be obtained as follows.

$$
F_{e}=\frac{d U_{e}}{d w_{\text {avg }}}=\frac{1}{2} V^{2} \frac{d C}{d w_{\text {avg }}}
$$

The pull-in voltage (8) can be computed at a critical stable displacement $\left(w_{\text {avg,PI }}\right)$, which is obtained by the methodology of energy stability criteria [37, 42].

$$
V_{P I}=\sqrt{2 F_{m}\left(w_{\text {avg }, P I}\right) /\left.\frac{d C}{d w_{\text {avg }}}\right|_{w_{\text {avg }}=w_{\text {avg }, P I}}}
$$

For the estimation of pull-in voltage $\left(V_{P I}\right)$ and stable peak displacement $\left(w_{\text {peak,PI}}\right)$ of the diaphragm center, the mechanical restoring force $\left(F_{m}\right)$ at a given displacement was retrieved by the multilayer nonlinear model. The voltage value, which allows the diaphragm to exist in equilibrium at a predefined displacement under combined effects of mechanical $\left(F_{m}\right)$ and electrical forces $\left(F_{e}\right)$, was estimated by the model formulation and validated by FEM analysis for the accuracy of the model. The numerical model was solved in COMSOL Multiphysics ( ) v5.3a by coupling Solid Mechanics and Electrostatics interfaces.

Figures 3(a) and (b) correspond to the required voltage, plotted against a sweep of the center displacement through the chamber depth $(g)$ for the diaphragms with variable thickness $\left(t_{3}\right)$. Again, a prediction error was defined to validate analytical voltage estimation by comparing it with the numerical results at the same predefined center displacement until the pull-in instability occurs. We provided the comparison plots in figures 3 (c) and (d) at initial gaps, $g=5 \mu \mathrm{m}$ and $g=15 \mu \mathrm{m}$. Analytically calculated voltages show reasonable accuracy with the numerical results. The percent error was below $7 \%$ for the investigated range of diaphragm thicknesses $\left(t_{3}\right)$.

Another significant observation is that the minimum values of stable peak displacement before pull-in instability are 0.732 of the initial gap for the diaphragm with $g=5 \mu \mathrm{m}$ and $t_{3}=5 \mu \mathrm{m}$, and 0.714 of the initial gap for the diaphragm with $g=15 \mu \mathrm{m}$ and $t_{3}=10 \mu \mathrm{m}$. These values are strictly comparable with the simulations $(0.726$ and 0.708 , respectively) and far beyond the travel range obtained by the simple parallel-plate capacitor approach, which corresponds to $1 / 3$ of the initial gap [36]. Moreover, the thick diaphragms $\left(t_{3}=20 \mu \mathrm{m}\right)$, depending on the initial gap $(g)$, can smoothly traverse over the entire gap with undergoing no pull-in discontinuity.

After validating the analytical model with the numerical results, we plotted the contours of the pull-in voltage of the diaphragms with different thickness $\left(t_{3}\right)$ for different initial gaps $(g)$. Figure 4 shows the corresponding design space of electrostatically actuated diaphragm with a $300 \mu \mathrm{m}$ radius. The contour plot enables the evaluation of the design feasibility to ensure operation within an acceptable range of potentials depending on the limit of available high voltage DC power supply, intended to drive the actuator.

\section{Fabrication}

The diaphragms were fabricated using standard MEMS fabrication methods and merged with prevalent microfluidic prototyping based on PDMS to enable the integration of microvalves into LOC devices. Figure 5 illustrates the complete fabrication flow, including definitions of the electrostatically actuated diaphragm and PDMS microchannel.

First, a glass wafer was sputtered with a bimetallic $\mathrm{Cr} / \mathrm{Au}$ $(50 \mathrm{~nm} / 500 \mathrm{~nm})$ layer to mask the glass for chemical etching. The locations of actuation chambers and air vent channels were defined by photolithography and patterned using the wet metal etch process. The glass wafer was etched in $49 \% \mathrm{HF}$ for $60 \mathrm{~s}$ to reach a chamber depth of $9 \mu \mathrm{m}$. After removing the photoresist (PR) mask and bimetallic layer, the wafer was subjected to a short buffered HF (1:7) treatment to smooth sharp pattern edges. Before the metallization of the bottom electrode, a $\mathrm{SiO}_{2}$ coating was realized by plasmaenhanced chemical vapor deposition (PECVD) to promote plasma bonding with PDMS at the microfluidic integration step.

As the next step, the bottom electrode was created by sputtering a chromium layer of $20 \mathrm{~nm}$ as an adhesion layer and then a gold layer of $200 \mathrm{~nm}$. The metal layers were patterned by a wet metal etch processes, after specifying the regions of the bottom electrodes, pads, and wires with photolithography. Following the definition of the electrode, a thin layer of parylene $(0.71 \mu \mathrm{m})$ was deposited with silane A-174 to increase its adhesion to the wafer surface (PDS 2010, SCS). The surface 


\section{(a) MEMS Fabrication of Electrostatic Diaphragms}

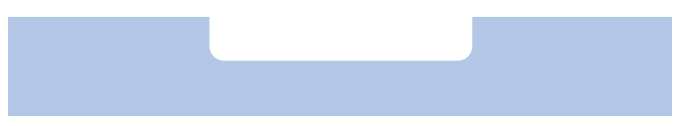

1. Etching glass with HF

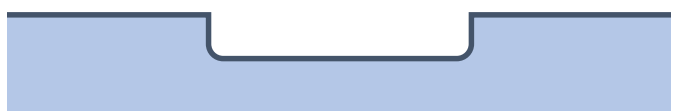

2. PECVD oxide deposition

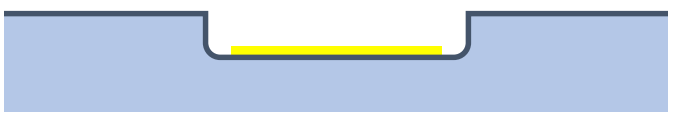

3. Bottom electrode metallization

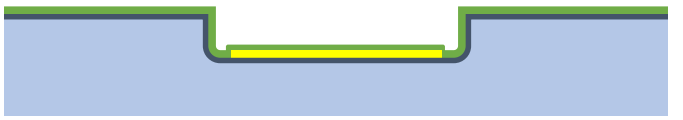

4. Parylene-C deposition with silane

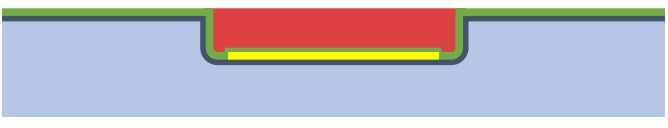

5. Filling of actuation chambers

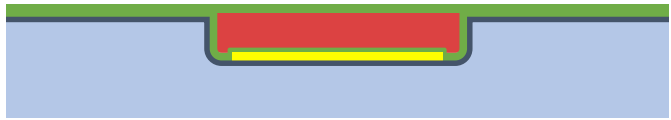

6. Parylene-C deposition for diaphragm definition

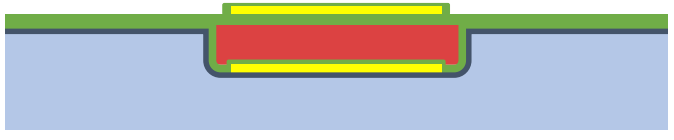

7. Top electrode metallization and parylene-C deposition for insulation

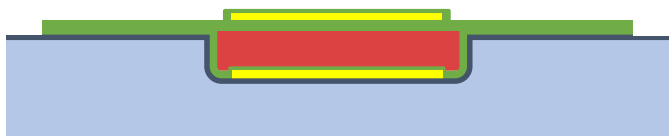

8. RIE etching of parylene-C

(b) Microchannel Fabrication and Sealing

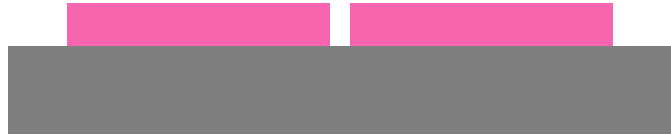

9. Mold fabrication

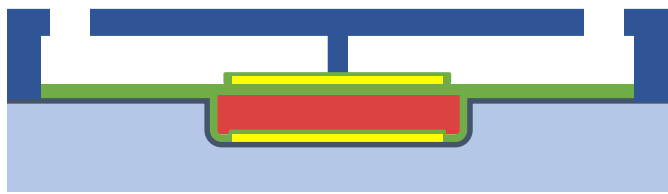

11. Sealing with plasma treatment

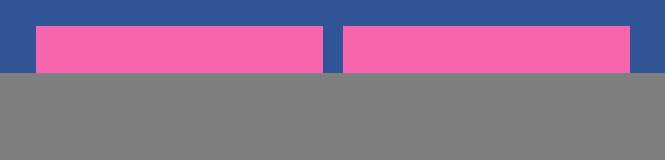

10. Pouring and curing of PDMS

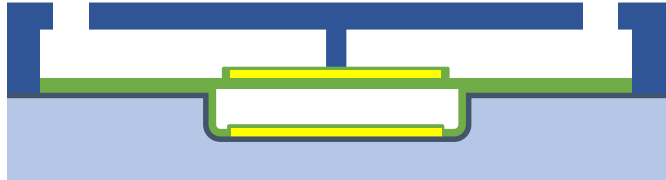

12. Release of the device

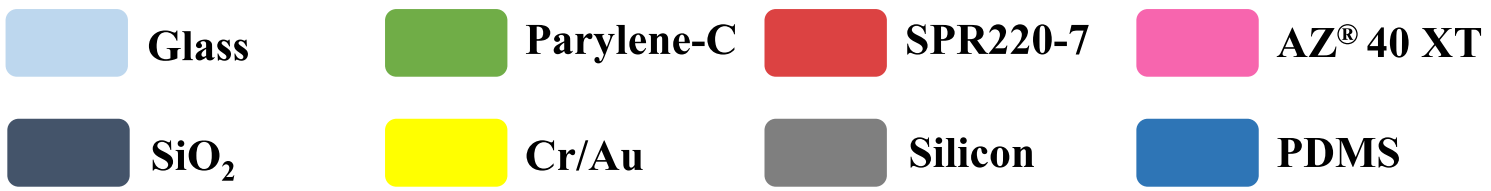

Figure 5. Fabrication flow of the electrostatic diaphragm (1-8) and microchannel definition (9-12).

stiction between two parylene-C layers is lower than that of parylene to gold or oxide surfaces when devices were air-dried during release [43]. This parylene layer ameliorates the stiction problem after releasing sacrificial PR and increases the dielectric strength of the electrostatic diaphragms.

Then, actuation chambers and air vent channels were filled with a sacrificial PR. We accurately adjusted the film thickness of PR attained by spin-coating to the depth of the actuation chamber. An eight-hour-long bake at $90{ }^{\circ} \mathrm{C}$ was applied to the wafer to ensure the endurance of the sacrificial PR during the following fabrication steps. A subnormal temperature was selected to preserve the integrity of the underlying Parylene$\mathrm{C}$ layer. A parylene layer of $3.79 \mu \mathrm{m}$ was deposited on top of the resist layer to form the diaphragm structure. Top electrode metallization $(\mathrm{Au} / \mathrm{Ti}, 200 \mathrm{~nm} / 20 \mathrm{~nm})$ was performed at low power $(100 \mathrm{~W})$ on parylene, and later, an additional thin parylene layer $(1.2 \mu \mathrm{m})$ was deposited on top of the electrode to prevent the electrolysis of working fluid.

After final parylene deposition, the surface parylene layer was removed by reactive ion etching (RIE) to define the diaphragm. During RIE, the parylene layer was preserved on the channel regions to increase endurance, and on metallic wires to provide electrical isolation. The diaphragms were released by immersing in acetone for two days and IPA for one day. After solvent treatment, the individual dies were air-dried and ready to be tested for the characterization of 

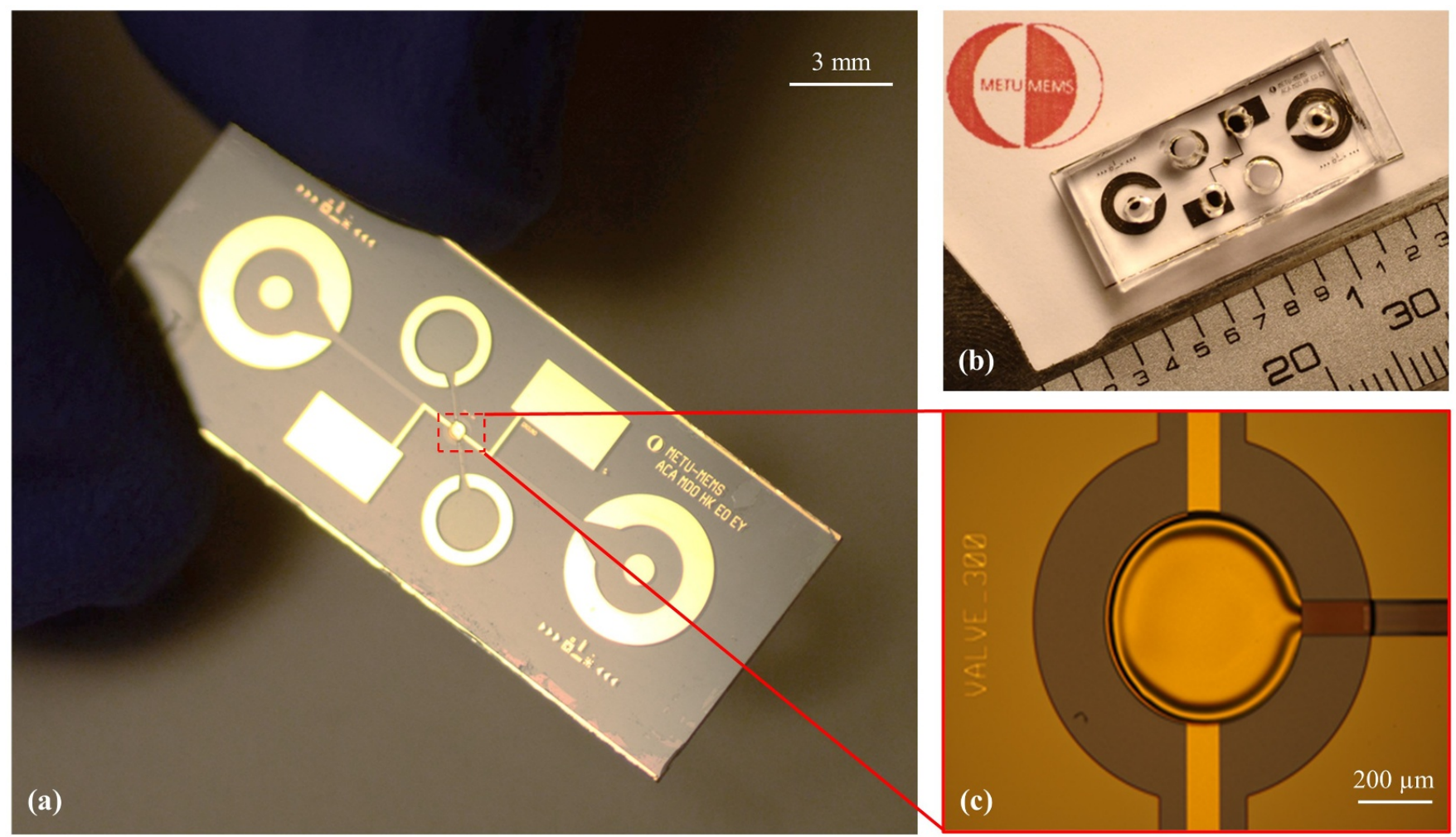

Figure 6. (a) The fabricated prototype of the electrostatically actuated diaphragm on the glass substrate. (b) The complete microvalve structure, constructed after plasma bonding. (c) A microscopic image (10x) depicting the diaphragm at the end of MEMS fabrication.

electrostatic diaphragms. Figures 6(a) and (c) show the fabricated diaphragm on the glass die and the microscopic image of the diaphragm, after completing dry etching of parylene, respectively.

The diaphragms can be integrated with a PDMS microchannel to realize a normally closed electrostatic microvalve. Thus, a silicon master was patterned with $50 \mu \mathrm{m}$-thick positive $\mathrm{AZ}^{\circledR}$ $40 \mathrm{XT}$ photoresist, which allows a good aspect ratio, to fabricate PDMS channels. In order to achieve easy peeling of PDMS from silicon mold by lowering the surface energy, we completed the silanization of the mold by keeping the wafer for a couple of hours in a vacuum desiccator together with a few drops of Trichloro(1 H,1 H,2 H,2H-perfluorooctyl)silane, 97\% (PFOTS, Sigma Aldrich). Then, PDMS prepolymer was mixed with the curing agent in a ratio of 10:1. The mixture was degassed in a desiccator, poured on the mold, and cured at room temperature to prevent significant shrinkage [44]. After the peeling of PDMS, the individual devices were cut by a razor blade, and inlet/outlet ports were opened using a biopsy punch.

Before plasma bonding, the surface of the glass was cleaned by immersing in acetone and IPA for $15 \mathrm{~min}$, respectively. The short cleaning treatment allows the sacrificial PR under diaphragm to preserve its integrity. So, the PR obviates the diaphragm stiction during sealing. The next step was the permanent bonding of these devices to create a tight seal for microchannel formation. We exposed both substrates to an oxygen plasma generated at $100 \mathrm{~W}$ for $90 \mathrm{sec}$ at die-level. Following the plasma treatment, a drop of liquid, which did not corrupt the plasma-activated surface such as methanol, ethanol, or water, was dropped between the glass and PDMS to allow enough time to achieve the alignment of devices. After contriving the alignment of two substrates under an optical microscope, they were put on a hot plate at $110{ }^{\circ} \mathrm{C}$ for $10 \mathrm{~min}$ to evaporate the liquid and permanently bond. More precise alignment and bonding can be achieved by building a simple adapter that can be connected to an optical microscope's objective for sophisticated microchannel networks [45]. Finally, the device was released by successively treating the prototypes by acetone and isopropyl alcohol and air-dried to be tested under fluid flow. Figure 6(b) illustrates a normally-closed electrostatically actuated PDMS-glass hybrid microvalve.

\section{Experimental validation}

During the tests for the electrical characterization of moving diaphragms, we used a high voltage supply to apply the necessary potential between the electrodes while observing under a probe station. Regarding videos and images were recorded via 25 fps camera and processed with ImageJ.

The characterization of electrostatic diaphragms fabricated on glass pertaining to pull-in voltage was completed under atmospheric pressure. In order to measure pull-in voltage, the applied voltage gradually increased by $1 \mathrm{~V}$-steps until diaphragm collapses to the bottom of the actuation chamber. The average pull-in voltage was measured around $221 \mathrm{~V}$ with $2.74 \mathrm{~V}$ deviation for four diaphragms with a $300 \mu \mathrm{m}$ radius. 

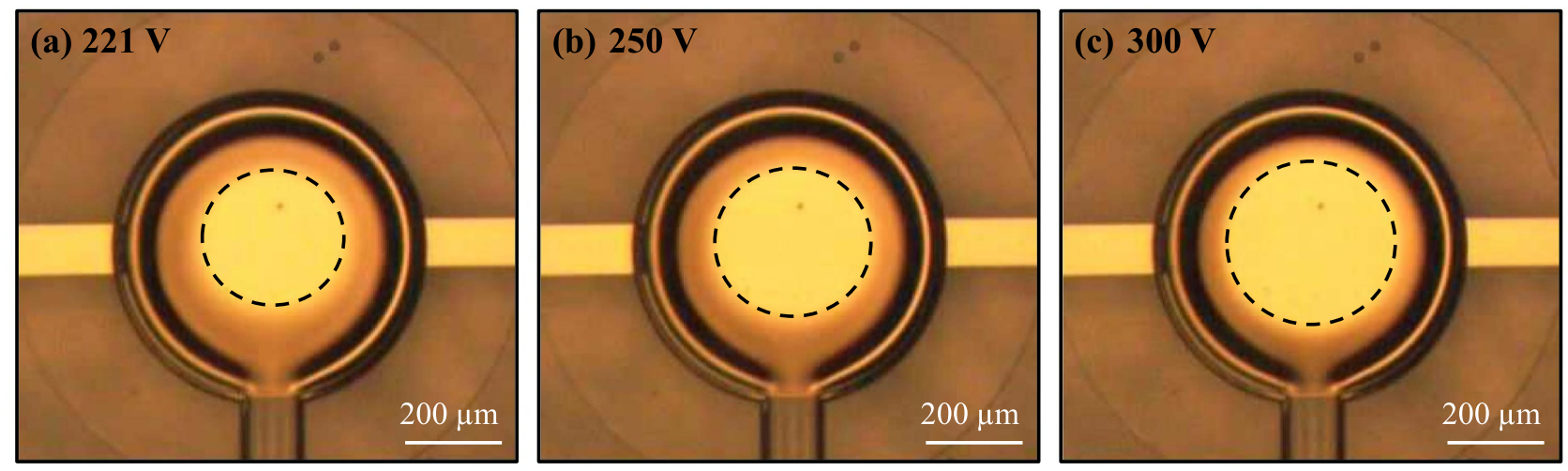

Figure 7. Snapshots of the diaphragm at different actuation potentials. The dashed circles denote the area touching to the bottom of the actuation chamber and were drawn at the outline of the segmented instances. (a) $V_{d c}=221 \mathrm{~V}$ (pull-in). (b) $V_{d c}=250 \mathrm{~V}$. (c) $V_{d c}=300 \mathrm{~V}$.

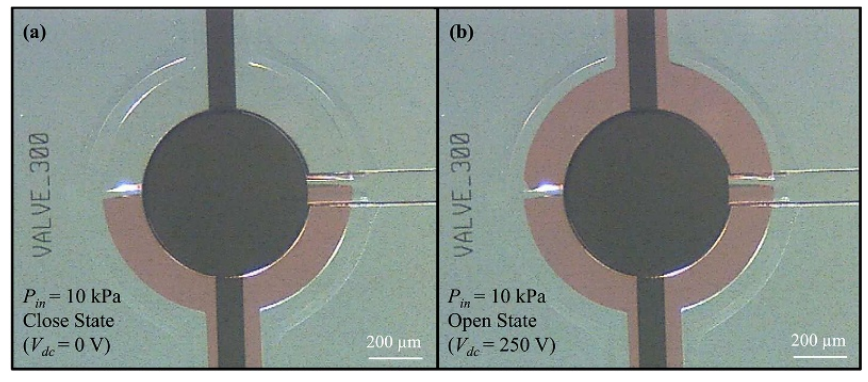

Figure 8. Microscopic images that indicate the burst mode operation of the electrostatic microvalve while working fluid is colored DI water [48]. (a) The fluid is given to the microchannel by applying positive pressure of $10 \mathrm{kPa}$ at the inlet. (b) The microvalve is actuated at $V_{d c}=250 \mathrm{~V}$.

Concerning parylene deposition thicknesses $\left(t_{1}=1.2 \mu \mathrm{m}\right.$, $\left.t_{3}=3.79 \mu \mathrm{m}, t_{4}=0.71 \mu \mathrm{m}\right)$ and the actuation chamber depth $(9.1 \mu \mathrm{m})$, following the fabrication, the expected values of pull-in voltage were estimated as $218.98 \mathrm{~V}$ and $223.03 \mathrm{~V}$ for numerical simulation and proposed analytical model, respectively. Thus, experimental values of pull-in voltage are in good agreement with both numerical and analytical studies and indicate the importance of including the geometric nonlinearities and calculating the stiffness for layered plates.

While going from open state to closed state, the diaphragms can respond to voltage variation in $70 \pm 17.32$ milliseconds when a $100 \mathrm{k} \Omega$ resistor was connected in parallel to discharge the actuator. However, the opening duration was greater than that of closing. The opening response time was $370 \pm 43.59$ milliseconds because the high voltage generator was unable to provide the desired potential abruptly. We extracted the response times by tracking the intermittent movement of the diaphragm frame-by-frame for four diaphragms, actuated at $250 \mathrm{~V}$. Moreover, the diaphragms were actuated periodically at $250 \mathrm{~V}$ more than 50 times, so the valving operation was repeatable.

After the pull-in, the applied potential was increased further to evaluate the touch area, which is the portion of the diaphragm contacting the base in touch-mode [46]. Figure 7 illustrates the snapshots of an actuated diaphragm while applying different potentials higher than pull-in voltage. In order to measure the area of a collapsed diaphragm, we employed the process of image segmentation. The corresponding image was first imported to ImageJ and converted to grayscale. A circular region of interest surrounding the inside of the diaphragm was defined. Outside of this region was cleared to obtain sharp intensity peaks in the histogram plot. By using histogram thresholding, the image was segmented into the feature of interest representing the touch area of the diaphragm. After thresholding, the area touching to the bottom of the actuation chamber was measured using the outline of the segmented instance. We used normalization to eliminate the spatial errors of the camera safely. Thus, the total diaphragm area was also extracted to normalize the measured touch areas, which have a scale unit of square pixels. The mean and variance of the normalized touch area were estimated using the Taylor series expansion of the ratio of two measured area variables for four different diaphragms [47]. The values of normalized areas at pull-in, $250 \mathrm{~V}$, and $300 \mathrm{~V}$ were $25.3 \% \pm 2.6 \%, 31.0 \% \pm 2.7 \%$, and $40.5 \% \pm 2.4 \%$, respectively. Thus, for a diaphragm with a $300 \mu \mathrm{m}$ radius, the touch area values at touch-mode can be calculated $0.0715 \pm 0.0074 \mathrm{~mm}^{2}, 0.0877 \pm 0.0076 \mathrm{~mm}^{2}$, and $0.1145 \pm 0.0068 \mathrm{~mm}^{2}$ at pull-in, $250 \mathrm{~V}$, and $300 \mathrm{~V}$, respectively. The investigation of the touch area beyond pull-in is essential for the determination of actuation voltage for different valve seat configurations, including the semicircular wall [40], or shifted wall [11].

Upon the electrical characterization of the electrostatic diaphragms under atmospheric pressure with no flow, we tested them under fluid flow (colored DI water) with a certain voltage applied, after irreversible sealing of PDMS channels onto the glass dies. The fluid flow was controlled by employing a microfluidic pressure controller (Fluigent, MFCS-EZ) at a positive pressure applied through the inlet port. At an applied pressure of $10 \mathrm{kPa}$, no leakage was observed. The liquid remains stationary due to the adhesion energy between the valve seat and the diaphragm (figure 8(a)). When the microvalve was actuated by applying $250 \mathrm{~V}$, working fluid flows through the valve region, indicating the burst mode operation by electrostatic actuation (figure 8(b)). The flow response concerning inlet pressure and actuation voltage was discussed previously by our research group for the proposed design [40]. For the valve reported here, we anticipate a better 
leakage behavior, because the thicker diaphragm will be stiffer and move less under the same fluidic pressure.

\section{Conclusion}

In this paper, we presented a nonlinear analytical model that accurately captures the pull-in voltage of electrostatically actuated diaphragms in conjunction with a multilayered plate formulation. The model can be used to assess the feasibility of the diaphragm design for the proposed microfluidic integration under specific operating voltage conditions. After having shared the model details, we thoroughly explained the hybrid fabrication scheme. The fabricated diaphragms were characterized for their operational performances, including the pull-in voltages, opening/closing response times, touch areas, and repeatability. Moreover, the diaphragm sealed with PDMS microchannel was tested under the flow of colored water to prove that the proposed channel integration allows fluid flow underneath the valve seat.

Guided by the analytical and numerical solution introduced in this work, several surface-micromachined binary PDMS-glass hybrid microvalves can be assembled on the same chip to work separately to achieve precision flow control on a microscopic level. Furthermore, the actuation chambers of the electrostatic microvalve arrays can be connected via a single venting network pneumatically supported from a single inlet to operate at high pressures without noticeable leakage. Moreover, the reported analytical expressions can also be utilized in the design of micro components such as pumps and pressure transducers, relying on the dynamics of electrostatic diaphragms.

The presented sealing method exploits a potential for the integration of on-chip electronics fabricated mostly on silicon or glass substrates with the polymer materials widely used in microfluidics to have integrated active elements. Despite all advantages of PDMS, it also has several fundamental limitations, such as poor swelling properties in certain organic solvents, relatively high vapor permeability, and absorption of small hydrophobic molecules. Hence, further research might be carried out to experiment possible integration of diaphragms with other organic polymers (e.g. PMMA, PC, COC), which can be irreversibly bonded to glass substrates through oxygen plasma treatment after surface activation with the use of a silane solution [49].

\section{Acknowledgments}

This work was funded by The Scientific and Technological Research Council of Turkey (TUBITAK) under grant number 114E098.

\section{ORCID iDs}

Ali Can Atik (iD https://orcid.org/0000-0002-1511-5527

Ebru Özgür (D) https://orcid.org/0000-0002-2469-0267

Haluk Külah (D) https://orcid.org/0000-0003-1331-4474
Ender Y1ldırım (D) https://orcid.org/0000-0002-7969-2243

\section{References}

[1] Manz A, Widmers H M and Graber N 1990 Miniaturized total chemical analysis systems: a novel concept for chemical sensing Sensors Actuators B $1244-8$

[2] Fu C, Rummler Z and Schomburg W 2003 Magnetically driven micro ball valves fabricated by multilayer adhesive film bonding J. Micromech. Microeng. 13 S96-102

[3] Bae B, Kim N, Kee H, Kim S H, Lee Y, Lee S and Park K 2002 Feasibility test of an electromagnetically driven valve actuator for glaucoma treatment J. Microelectromech. Syst. $11344-54$

[4] Hilbich D D, Khosla A, Gray B L and Shannon L 2011 Bidirectional magnetic microactuators for uTAS Proc. SPIE $792979290 \mathrm{H}$

[5] Yamahata C, Lacharme F, Matter J, Schnydrig S, Burri Y and Gijs M A M 2005 Electromagnetically actuated ball valve micropumps The 13th Int. Conf. on Solid-State Sensors, Actuators and Microsystems vol 1 pp 192-6

[6] Meckes A, Behrens J, Kayser O, Benecke W, Becker T and Müller G 1999 Microfluidic system for the integration and cyclic operation of gas sensors Sensors Actuators A $76478-83$

[7] Casals-Terré J, Duch M, Plaza J A, Esteve J, Pérez-Castillejos R, Vallés E and Gómez E 2008 Design, fabrication and characterization of an externally actuated ON/OFF microvalve Sensors Actuators A 147 600-6

[8] Bintoro J S, Hesketh P J and Berthelot Y H 2005 CMOS compatible bistable electromagnetic microvalve on a single wafer Microelectron. J. 36 667-72

[9] Yoshida K, Tanaka S, Hagihara Y, Tomonari S and Esashi M 2010 Normally closed electrostatic microvalve with pressure balance mechanism for portable fuel cell application Sensors Actuators A 157 290-8

[10] Patrascu M, Gonzalo-Ruiz J, Goedbloed M, Brongersma S H and Crego-Calama M 2012 Flexible, electrostatic microfluidic actuators based on thin film fabrication Sensors Actuators A 186 249-56

[11] Tice J D, Rosheck J B, Hamlin C D, Apblett C A and Kenis P J A 2013 Normally-closed electrostatic microvalve fabricated using exclusively soft-lithographic techniques and operated with portable electronics J. Microelectromech. Syst. 22 1251-3

[12] Desai A V, Tice J D, Apblett C A and Kenis P J A 2012 Design considerations for electrostatic microvalves with applications in poly(dimethylsiloxane)-based microfluidics Lab. Chip. 12 1078-88

[13] Liu X and Li S 2014 An electromagnetic microvalve for pneumatic control of microfluidic systems J. Lab. Autom. 19 444-53

[14] Pekas N, Zhang Q and Juncker D 2012 Electrostatic actuator with liquid metal-elastomer compliant electrodes used for on-chip microvalving J. Micromech. Microeng. 22097001

[15] Shao P, Rummler Z and Schomburg W K 2004 Polymer micro piezo valve with a small dead volume $J$. Micromech. Microeng. 14 305-9

[16] Evans A T, Park J M, Chiravuri S and Gianchandani Y B 2010 A low power, microvalve regulated architecture for drug delivery systems Biomed. Microdevices 12 159-68

[17] Cazorla P-H, Fuchs O, Cochet M, Maubert S, Le Rhun G, Fouillet Y and Defay E 2014 Integration of PZT thin films on a microfluidic complex system 2014 IEEE Int. Ultrasonics Symp pp 491-4

[18] Roberts D C, Li H, Steyn L, Yaglioglu O, Spearing S M Schmidt M A and Hagood N W 2003 A piezoelectric microvalve for compact high-frequency, high-differential 
pressure hydraulic micropumping systems $J$.

Microelectromech. Syst. 12 81-92

[19] Li H Q, Roberts D C, Steyn J L, Turner K T, Yaglioglu O, Hagood N W, Spearing S M and Schmidt M A 2004 Fabrication of a high frequency piezoelectric microvalve Sensors Actuators A 111 51-56

[20] Barth P W 1995 Silicon microvalves for gas flow control Proc. of the Int. Solid-State Sensors and Actuators Conf. vol 2 pp 276-9

[21] Rich C A and Wise K D 2003 A high-flow thermopneumatic microvalve with improved efficiency and integrated state sensing J. Microelectromech. Syst. 12 201-8

[22] Takao H, Miyamura K, Ebi H, Ashiki M, Sawada K and Ishida M 2005 A MEMS microvalve with PDMS diaphragm and two-chamber configuration of thermo-pneumatic actuator for integrated blood test system on silicon Sensors Actuators A 119 468-75

[23] Kohl M, Dittmann D, Quandt E and Winzek B 2000 Thin film shape memory microvalves with adjustable operation temperature Sensors Actuators A 83 214-9

[24] Liu Y, Kohl M, Okutsu K and Miyazaki S 2004 A TiNiPd thin film microvalve for high temperature applications Mater. Sci. Eng. A 378 205-9

[25] Wang Y-C, Choi M H and Han J 2004 Two-dimensional protein separation with advanced sample and buffer isolation using microfluidic valves Anal. Chem. 76 4426-31

[26] Hosokawa K and Maeda R 2000 A pneumatically-actuated three-way microvalve fabricated with polydimethylsiloxane using the membrane transfer technique J. Micromech. Microeng. 10 415-20

[27] Grover W H, Skelley A M, Liu C N, Lagally E T and Mathies R A 2003 Monolithic membrane valves and diaphragm pumps for practical large-scale integration into glass microfluidic devices Sensors Actuators B 89 315-23

[28] Ohori T, Shoji S, Miura K and Yotsumoto A 1998 Partly disposable three-way microvalve for a medical micro total analysis system ( $\mu$ TAS) Sensors Actuators A 64 57-62

[29] Addae-Mensah K A, Cheung Y K, Fekete V, Rendely M S and Sia S K 2010 Actuation of elastomeric microvalves in point-of-care settings using handheld, battery-powered instrumentation Lab. Chip. 10 1618-22

[30] Unger M A, Unger M A, Chou H, Thorsen T, Scherer A and Quake S R 2013 Monolithic microfabricated valves and pumps by multilayer soft lithography Science 288 113-6

[31] Oh K W and Ahn C H 2006 A review of microvalves $J$. Micromech. Microeng. 16 R13-39

[32] Au A K, Lai H, Utela B R and Folch A 2011 Microvalves and micropumps for BioMEMS Micromachines 2 179-220

[33] Saheb J F, Richard J F, Meingan R, Sawan M and Savaria Y 2005 System integration of high voltage electrostatic MEMS actuators 3rd Int. IEEE-NEWCAS Conf. 2005 pp 155-8

[34] Zhang Y and Zhao Y P 2006 Numerical and analytical study on the pull-in instability of micro-structure under electrostatic loading Sensors Actuators A 127 366-80
[35] Abdel-Rahman E M, Younis M I and Nayfeh A H 2002 Characterization of the mechanical behavior of an electrically actuated microbeam J. Micromech. Microeng. $12759-66$

[36] Hung E S and Senturia S D 1999 Extending the travel range of analog-tuned electrostatic actuators J. Microelectromech. Syst. 8 497-505

[37] Wygant I O, Kupnik M and Khuri-Yakub B T 2008 Analytically calculating membrane displacement and the equivalent circuit model of a circular CMUT cell 2008 IEEE Ultrasonics Symp. pp 2111-4

[38] Chang M P and Maharbiz M M 2009 Electrostatically-driven elastomer components for user-reconfigurable high density microfluidics Lab. Chip. 9 1274-81

[39] Sounart T L, Michalske T A and Zavadil K R 2005 Frequency-dependent electrostatic actuation in microfluidic MEMS J. Microelectromech. Syst. 14 125-33

[40] Yildirim E, Arikan M A S and Külah H 2012 A normally closed electrostatic parylene microvalve for micro total analysis systems Sensors Actuators A 181 $81-86$

[41] Timoshenko S and Woinowski-Krieger S 1959 Theory of Plates and Shells (New York: McGraw-Hill)

[42] Nemirovsky Y and Bochobza-Degani O 2001 A methodology and model for the pull-in parameters of electrostatic actuators J. Microelectromech. Syst. 10 601-15

[43] Feiqiao Y, Lin J C-H, Chen P-J and Tai Y-C 2010 Stiction of parylene $\mathrm{C}$ to silicon surface measured using blister tests 2010 IEEE 5th Int. Conf. on Nano/Micro Engineered and Molecular Systems vol 2 pp 313-6

[44] Madsen M H, Feidenhans'I N A, Hansen P-E, Garnæs J and Dirscherl K 2014 Accounting for PDMS shrinkage when replicating structures J. Micromech. Microeng. 24127002

[45] Sivakumarasamy R, Nishiguchi K, Fujiwara A, Vuillaume D and Clément N 2014 A simple and inexpensive technique for PDMS/silicon chip alignment with sub- $\mu \mathrm{m}$ precision Anal. Methods 6 97-101

[46] Yıldırım E and Külah H 2011 Analysis and characterization of an electrostatically actuated in-plane parylene microvalve $J$. Micromech. Microeng. 21105009

[47] van Kempen G M P and van Vliet L J 2000 Mean and variance of ratio estimators used in fluorescence ratio imaging Cytometry 39 300-5 (https://onlinelibrary. wiley.com/doi/full/10.1002/\%28SICI\%291097$0320 \% 2820000401 \% 2939 \% 3 \mathrm{~A} 4 \%$ 3C300\%3A\%3AAID-CYTO8\%3E3.0.CO\% $3 \mathrm{~B} 2-\mathrm{O})$

[48] Atik A C 2019 Development of an Integrated Lab-on-a-chip (LOC) Platform for Multidrug Effect Analysis MSc Thesis Middle East Technical University

[49] Vlachopoulou M-E, Tserepi A, Pavli P, Argitis P, Sanopoulou $\mathrm{M}$ and Misiakos K 2009 A low temperature surface modification assisted method for bonding plastic substrates J. Micromech. Microeng. 19015007 